
\title{
28 Research Suare \\ Usefulness of Automated Image Analysis for Recognition of the Fragile X Syndrome Gestalt in Congolese Subjects
}

Toni Kasole Lubala

University of Lubumbashi

Tony Kayembe-Kitenge ( $\nabla$ tonykayemb@gmail.com )

Higher Institute of Medical Techniques

Gerrye Mubungu

University of Kinshasa

Aimé Lumaka

University of Kinshasa

Gray Kanteng

University of Lubumbashi

Sarah Savage

FDNA Inc

Oscar Luboya

Higher Institute of Medical Techniques

\section{Randi Hagerman}

University of California Davis Medical Center

Koenraad Devriendt

University Hospital Leuven, University of Leuven

Prosper Lukusa-Tshilobo

University Hospital Leuven, University of Leuven

\section{Research Article}

Keywords: Face2Gene, Fragile X syndrome, Screening, DR Congo

Posted Date: December 16th, 2021

DOI: https://doi.org/10.21203/rs.3.rs-1082768/v1

License: (a) (i) This work is licensed under a Creative Commons Attribution 4.0 International License.

Read Full License 


\section{Abstract \\ Background}

Computer-aided software such as the facial image diagnostic aid (FIDA) and Face2Gene has been developed to perform pattern recognition of facial features with promising clinical results. The aim of this study was to test Face2Gene's recognition performance on Bantu Congolese subjects with Fragile $X$ syndrome (FXS) as compared to Congolese subjects with intellectual disability but without FXS (nonFXS).

\section{Method}

Frontal facial photograph from 156 participants (14 patients with FXS and 142 controls) were uploaded. Automated face analysis was conducted by using the technology used in proprietary software tools called Face2Gene CLINIC and Face2Gene RESEARCH (version 17.6.2). To estimate the statistical power of the Face2Gene technology in distinguishing affected individuals from controls, a cross validation scheme was used.

\section{Results}

The similarity seen in the upper facial region (of males and females) is greater than the similarity seen in other parts of the face. Binary comparison of FXS subjects versus subjects with ID negative for Fragile $X$ syndrome and FXS subjects versus subjects with Down syndrome reveal an area under the curve values of $0.955(p=0.002)$ and $0.986(p=0.003)$.

\section{Conclusion}

The Face2Gene algorithm is separating well between FXS and Non-FXS subjects.

\section{Introduction}

Fragile X Syndrome (FXS) is a leading genetic cause of inherited intellectual disability and autism spectrum disorders (ASD) worldwide ${ }^{1}$. Despite the success in the molecular diagnosis of the FXS, challenges remain in designing a simple and effective tool to aid in the diagnosis in a clinical setting. At least eight clinical checklists have been developed for preselection of subjects based on clinical features $^{2-7}$. They have greatly improved the cost-effectiveness of Fragile-X testing by significantly reducing of the number of individuals to be tested ${ }^{8}$. However, their sensitivity remains a limitation to their application as a first-line screening test. 
Over the last decade, significant improvements in automated image analysis have been achieved. Computer-aided software such as the facial image diagnostic aid (FIDA) and Face2Gene has been developed to perform pattern recognition of facial features with promising clinical results. Face2Gene (FDNA, Inc., Boston, MA) offers the potential for a universal, rapid, and inexpensive aid to syndrome diagnosis $^{9}$ as it is designed to be used on computers and smartphones. Our aim was to test Face2Gene's

recognition performance on Bantu Congolese subjects with FXS as compared to Congolese subjects with intellectual disability (ID) but without FXS (non-FXS) and to quantify the ability of the technology to discriminate between these groups based on facial images analysis.

\section{Patients And Controls}

Frontal photographs of 156 participants (14 patients with FXS and 142 controls with ID) of Bantu Congolese origin, were identified in Lubumbashi and Kinshasa from March 2014 to March 2018. FXS was diagnosed by DNA molecular analysis.

Subjects included in the FXS group all had a full mutation (8 Males; 6 Females; Median Age: 18.9 years; Range 4-39). Two separate control groups of Congolese non-FXS subjects, either with intellectual disability of unknown origin or with Down syndrome, were included. The first test set included 100 Congolese subjects with intellectual disability but negative for Fragile $X$ syndrome ( 68 Males and 32 Females) aged between 5 and 40 years (median 17). In the second test set, we recruited 42 subjects with Down syndrome (29 males and 13 Females) age 6 to 40 (median 15.34).

\section{Fragile $X$ syndrome genetic testing}

Patient's genomic DNA was isolated using standard protocols from the peripheral blood leukocytes. A combination of long-range PCR and triplet repeat primed PCR (TP-PCR) using the AmplideX® PCR/CE FMR1 Reagents kit (Asuragen, www.asuragen.com) was performed, with size differentiation on the Applied Biosystems 3130xI Genetic Analyzer to test for the FMR1 expansion. Mutations have been classified according to GenBank NM_002024.5.

\section{Picture analysis computed evaluation of Congolese subjects}

Automated face analysis was conducted using the technology from the proprietary software tools called Face2Gene CLINIC and Face2Gene RESEARCH (version 17.6.2). This technology combines facial recognition software with clinical feature annotation using Human Phenotype Ontology ${ }^{10}$ and anthropometric measurements. The software enables detection of recognizable patterns of human malformations from two-dimensional facial photographs, despite individual differences in variables such as ethnicity, sex, and age. The analysis of visual facial data is used to form a mathematical representation of the face (facial descriptor) which can be readily compared to other such descriptors ${ }^{9}$.

Patient images were analyzed using the Face2Gene CLINIC tool in order to assess the technology's ability to determine whether FXS is a relevant syndrome suggestion. We compared the tool's performance for 
FXS subjects and non-FXS subjects of Bantu Congolese ancestry. Cohorts for the phenotypic comparison were based on the clinical features and/or molecular diagnosis. Patients' facial descriptors were compared to syndrome gestalts to quantify similarity (gestalt scores), resulting in a prioritized list of syndromes with similar morphology. The algorithm currently in use at the time of the analysis utilized a FXS facial model that was previously trained by the software programmers based on a total of 111 cases. Of these, 60 were listed as Caucasian, 14 were listed as African, 20 listed as African American, and 2 listed as Asian.

Patient images were also analyzed using the Face2Gene RESEARCH tool in order to conduct a statistical comparison between facial patterns of each of the three cohorts: FXS subjects, non-FXS subject with ID, and subject with DS. The individual facial descriptors for the patients in each defined cohort were used to train and then test a novel facial model for each cohort, using a binary comparison approach. This enabled a statistical analysis of the technology's ability to accurately distinguish between all cohorts.

\section{Picture acquisition conditions}

Only a frontal facial photograph was uploaded, and no further clinical features (HPO phenotypes) were annotated. Therefore, the differential diagnoses provided represent syndromes that achieved the highest gestalt scores in the image analysis and do not depend on clinical findings identified. Digital photographs of the patients' faces were taken with a resolution of $640 \times 480$ pixel in frontal pose in front of a white or grey background with the Nikon Coolpix 950 camera.

\section{Statistics}

In the present study, specificity, sensitivity, as well as positive and negative predictive value are expressed as percentages. Confidence intervals for sensitivity, specificity and accuracy are "exact" Clopper-Pearson confidence intervals. Confidence intervals for the likelihood ratios are calculated using the "Log method" 11,12 . Confidence intervals for the predictive values are the standard logit confidence intervals ${ }^{13}$.

To estimate the statistical power of the Face2Gene technology in distinguishing affected individuals from controls, a cross validation scheme was used, including a series of binary comparisons between all groups. For these binary comparisons, the data was split randomly multiple times into training sets and test sets. Each such set contained half of the samples from the group, and this random process was repeated 10 times.

The comparison and separation quality between all three groups was evaluated by measuring the Area Under the Curve (AUC) of the Receiver Operating Characteristic (ROC) curve. The ROC curve is a widely accepted form of measuring classification success. Results for our experiment are reported by computing the area under the curve (AUC) statistic of the ROC curve. An AUC of 1 is indicative of perfect accuracy, whereas an AUC of 0.5 is the equivalent performance obtained by a completely random coin-flipping system $(0.80-0.90=$ good accuracy; $0.70-0.80=$ fair accuracy and $0.60-0.70=$ poor $)$. The facial descriptor can also be graphically displayed as a two-dimensional model of the face (mask) specific to the 
particular condition of interest. These two-dimensional masks can be used to visually represent the comparison of two digital facial descriptors, and a graphical heatmap can be applied to visualize the degree of similarity between the two descriptors being compared. In this article these above techniques were used.

\section{Ethics}

For each participant with FXS, parents or legal representative provided written consent for study participation with an informed consent that was approved with our protocol by the ethical committee of the University of Lubumbashi (UNILU/CEM/027/2018). The study methods were performed in accordance with guidelines and regulations. We applied an anonymous and non-personal coding system to protect participants' privacy. The study participants are patients that are included in the follow up program that we are doing, we obtained the consent to publish these data from parents and/or tutors of study participants.

\section{Results}

After analysis in Face2Gene CLINIC, FXS was listed in the top 10 syndrome-matches for all 14 subjects with FXS (100\% sensitivity), whereas FXS was suggested for 47 out of 100 non-FXS subjects (53\% specificity), with an accuracy of $58.77 \%$ (Table 1 ).

Heat-maps display the level of similarity between the patients' photograph and the syndrome mask. As shown in Figure 1, the similarity we observe in our 14 FXS subjects indicates higher in the upper facial region than in other parts of the face (Figure 1).

FXS was listed in the top 10 syndrome-matches for all 14 subjects with FXS (100\% sensitivity, whereas 47 non-FXS subjects were recognized as FXS (53\% specificity) with an accuracy of $58.77 \%$ (table 1 ).

Binary comparison of FXS subjects versus subjects with ID and negative for FXS shows an area under the curve value of $0.955(p=0.002)$ (Figure $2 A)$. Comparison of FXS subjects versus subjects with Down syndrome shows and area under the curve of $0.986(p=0.003)$ (Figure 2B).

\section{Discussion}

In the present study, we used two approaches to evaluate how well the technology in Face2Gene discriminates between Bantu Congolese subjects with intellectual disability with different underlying diagnoses. We tested the Face2Gene tool to compare its performance in recognition of Bantu Congolese subjects with FXS and to discriminate this from other causes of ID. To estimate the statistical power of the Face2Gene Technology in distinguishing FXS individuals from non-FXS ID, a cross validation scheme was used, including a series of binary comparisons between the 2 groups. The binary comparison results demonstrate a high discrimination between those with FXS and the control groups, non-FXS ID and Down syndrome. 
FXS was listed in the top 10 syndrome-matches for all 14 subjects with FXS (100\% sensitivity). In contrast, FXS was included in the top 10 syndrome matches for 47 non-FXS subjects (53,0\% specificity) which results in an accuracy of $58.8 \%$.

A limitation of this study is the small number of cases studied. If confirmed in a larger population, this high sensitivity may mean that absence of FXS in the first 10 matches renders this diagnosis unlikely, and genetic testing of the FMR1 gene has a low priority.

Due to the high sensitivity achieved, Face2Gene promises to be a valid tool for large first-line test of a sequential screening strategy for FXS high-risk populations. After this first screening is conducted, subjects who test positive can be reevaluated for a second test such as the clinical universal checklist to further reduce false positives ${ }^{13}$. Consequently, when positives from the first-line test are retested, there is a net loss in sensitivity but a net gain in specificity. Moreover, Face2Gene has the double advantage of reproducibility, repeatability and reliability of its outcomes and the absence of inter and intra-observer variations.

Heat-maps of subjects in the FXS group display the level of similitude between the patient photo and syndrome mask, mostly in the upper part of the face. FXS subjects are known to display high and large quadrangular forehead, prominent supraorbital ridges and relative macrocephaly. Recently, a structural longitudinal magnetic resonance imaging study of preschoolers with FXS observed generalized brain overgrowth in children with FXS compared with controls, which become evident before the complete fusion of the skull bones by the ossification of sutural growth centers. Additionally, the decreased width of the inner skull has previously been described in fragile $X$ mice $^{14}$. This observation seems to correlate with the hypothesis that the macrocephaly and large forehead that have been observed in FXS subjects may be a consequence of the underlying global brain overgrowth. Moreover, the impression of large upper part of the face may also be a consequence of the extreme narrowing of the mid-face (bizygomatic and bigonial narrowing) that can be seen especially in full mutation males and have been extensively described in Heulens et al study using 3D facial photographs of a large cohort of FXS subjects. Another hypothesis states that craniofacial malformations observed in FXS can be caused by defects in the proliferation, migration or differentiation of pluripotent cranial neural crest cells that are involved in the formation of the cranial skeleton due to FMR1 gene silencing ${ }^{14}$. The broad forehead appears, therefore, to be a universal dysmorphic feature in subjects with FXS because the upper region of the face is less likely to be affected by ethnicity than periorbital, nasal and peri buccal regions that have been told to vary significantly among subjects according to their race. ${ }^{15-21}$. As it has been shown thus far, there are certain features that are restricted or more prominent in patients according to their ethnic background. Indeed, population inherent differences in dysmorphic features among children with Fetal Alcohol Spectrum Disorders have been found in a recent multiethnic study. The South African controls have been shown to display higher rates of narrow vermillion border, smooth philtrum, short interpupillary distance, flat nasal bridge, and anteverted nostrils, while control subjects from Indian ethnic background had higher 
rates of "railroad track" ears. Italian controls, however, fall between the 2 groups in most categories with the exception of philtrum length and strabismus ${ }^{22,23}$.

The overall older age of the studied patients was a limitation for the present study. Early diagnosis is crucial in genetic counselling of young parents, who may wish other children. The facial features in FXS become more prominent with age ${ }^{14}$. Therefore, studies in younger children are required to demonstrate the usefulness of this tool to diagnose accurately FXS in young children.

This technology is accessible and may be useful to clinicians particularly in developing countries where access to molecular testing is limited.

\section{Declarations}

Author Contributions: Conceptualization, T.KL, and O.L; data curation, T.K.-K., G.M.; G.K. and A.L; formal analysis, T.KL. T.K-K and S.S.; field work: T.KL; T.K.-K., G.K.; G.M.; methodology, K.D.; R.H.; L.T.KL; A.L.; and S.S.; software, T.KL. G.K.; A.L. and R.H.; supervision, O.L. and L.T; writing-original draft, T.KL; Writingreview and editing, T. KL. T.K.-K., G.M., A.L., G.K., S.S., O.L., R.H., K.D., and L.T. All authors have read and agreed to the published version of the manuscript

\section{Acknowledgements}

We would like to thank Jean Vanderpas, Benoit Nemery and Nicole Fleisher for their kind assistance during the redaction of this manuscript.

\section{Funding: No}

\section{CONFLICT OF INTEREST}

The authors declare no potential conflict of interest.

\section{DATA AVAILABILITY STATEMENT}

The data that support the findings of this study are available from the corresponding author upon reasonable request.

\section{References}

1. Gallagher, A. \& Hallahan, B. Fragile X-associated disorders: a clinical overview. J Neurol, 259 (3), 401-413 https://doi.org/doi:10.1007/s00415-011-6161-3 (2012).

2. Butler, G., Fennell, M., Robson, P. \& Gelder, M. Comparison of behavior therapy and cognitive behavior therapy in the treatment of generalized anxiety disorder. J Consult Clin Psychol, 59 (1), 167-175 https://doi.org/doi:10.1037//0022-006x.59.1.167 (1991). 
3. Hagerman, R. J., Amiri, K. \& Cronister, A. Fragile X checklist. Am J Med Genet, 38 (2-3), 283-287 https://doi.org/doi:10.1002/ajmg.1320380223 (1991).

4. Guruju, M. R. et al. Assessment of a clinical checklist in the diagnosis of fragile X syndrome in India. J Clin Neurosci Off J Neurosurg Soc Australas, 16 (10), 1305-1310 https://doi.org/doi:10.1016/j.jocn.2008.12.018 (2009).

5. Giangreco, C. A., Steele, M. W., Aston, C. E., Cummins, J. H. \& Wenger, S. L. A simplified six-item checklist for screening for fragile $X$ syndrome in the pediatric population. J Pediatr, 129 (4), 611-614 https://doi.org/doi:10.1016/s0022-3476(96)70130-0 (1996).

6. Laing, S., Partington, M., Robinson, H. \& Turner, G. Clinical screening score for the fragile X (MartinBell) syndrome. Am J Med Genet, 38 (2-3), 256-259 https://doi.org/doi:10.1002/ajmg.1320380219 (1991).

7. Limprasert, P. et al. A clinical checklist for fragile X syndrome: screening of Thai boys with developmental delay of unknown cause. J Med Assoc Thail Chotmaihet Thangphaet, 83 (10), 12601266 (2000).

8. Mandel, J-L. \& Chelly, J. Monogenic X-linked mental retardation: is it as frequent as currently estimated? The paradox of the ARX (Aristaless X) mutations. Eur J Hum Genet EJHG, 12 (9), 689693 https://doi.org/doi:10.1038/sj.ejhg.5201247 (2004).

9. Gurovich, Y. et al. Identifying facial phenotypes of genetic disorders using deep learning. Nat Med, 25 (1), 60-64 https://doi.org/doi:10.1038/s41591-018-0279-0 (2019).

10. Köhler, S. et al. Expansion of the Human Phenotype Ontology (HPO) knowledge base and resources. Nucleic Acids Res, 47 (D1), D1018-D1027 https://doi.org/doi:10.1093/nar/gky1105 (2019).

11. Altman, D. G. \& Royston, P. What do we mean by validating a prognostic model? Stat Med, 19 (4), 453-473 https://doi.org/doi:10.1002/(sici)1097-0258(20000229)19:4<453::aid-sim350>3.0.co;2-5 (2000).

12. Altman, D. G. Statistics in medical journals: some recent trends. Stat Med, 19 (23), 3275-3289 https://doi.org/doi:10.1002/1097-0258(20001215)19:23<3275::aid-sim626>3.0.co;2-m (2000).

13. Lubala, K. et al. Fragile X Checkl Metaanalysis Dev Simpl Univers Clin Checkl. 2018;(0):1-7. doi:https://doi.org/10.1002/mgg3.398 |

14. Heulens, I. et al. Craniofacial characteristics of fragile X syndrome in mouse and man. Eur J Hum Genet EJHG, 21 (8), 816-823 https://doi.org/doi:10.1038/ejhg.2012.265 (2013).

15. Tahmasebi, F., Khanehzad, M., Madadi, S. \& Hassanzadeh, G. Anthropometric Study of Nasal Parameters in Iranian University Students. Anat Sci J, 12 (4), 167-170 (2015).

16. Akinlolu, A. A., Akinola, B. O., Nurudeen, R. L., Kadiri, R. E. \& Ajao, M. S. Cephalometric Study of Mouth Morphology among Major Nigerian Tribes. Anat J Afr, 4 (1), 496-504 (2015).

17. Asghar, A., Dixit, A. \& Rani, M. Morphometric Study of Nasal Bone and Piriform Aperture in Human Dry Skull of Indian Origin. J Clin Diagn Res JCDR, 10 (1), AC05-AC07 https://doi.org/doi:10.7860/JCDR/2016/15677.7148 (2016). 
18. Hwang, T-S. \& Kang, H-S. Morphometry of nasal bases and nostrils in koreans. Ann Anat - Anat Anz, 185 (2), 189-193 https://doi.org/doi:10.1016/S0940-9602(03)80088-2 (2003).

19. Abdulrasheed, I. \& Eneye, A. M. Philtral Columns and Nostril Shapes in Nigerian Children: A Morphometric and Aesthetic Analysis. Plast Surg Int. 2013;2013. doi:10.1155/2013/382754

20. Quant, J. R. \& Woo, G. C. Normal values of eye position in the Chinese population of Hong Kong. Optom Vis Sci Off Publ Am Acad Optom, 69 (2), 152-158 (1992).

21. Price, K. M., Gupta, P. K., Woodward, J. A., Stinnett, S. S. \& Murchison, A. P. Eyebrow and eyelid dimensions: an anthropometric analysis of African Americans and Caucasians. Plast Reconstr Surg, 124 (2), 615-623 https://doi.org/doi:10.1097/PRS.0b013e3181addc98 (2009).

22. Lumaka, A. et al. Facial dysmorphism is influenced by ethnic background of the patient and of the evaluator. Clin Genet, 92 (2), 166-171 https://doi.org/doi:10.1111/cge.12948 (2017).

23. May, P. A. et al. Population differences in dysmorphic features among children with fetal alcohol spectrum disorders. J Dev Behav Pediatr JDBP, 31 (4), 304-316 https://doi.org/doi:10.1097/DBP.0b013e3181dae243 (2010).

\section{Tables}

Table I: Derived sensitivities, specificities, and predictive values for the Fragile $X$ syndrome using Face2Gene Algorithm 


\begin{tabular}{|c|c|c|c|c|c|}
\hline Test & Present & $\mathrm{n}$ & Absent & $\mathrm{n}$ & Total \\
\hline Positive & True Positive & 14 & $\begin{array}{l}\text { False } \\
\text { Positive }\end{array}$ & 47 & 61 \\
\hline Negative & $\begin{array}{l}\text { False } \\
\text { Negative }\end{array}$ & 0 & $\begin{array}{l}\text { True } \\
\text { Negative }\end{array}$ & 53 & 53 \\
\hline Total & & 14 & & 100 & 114 \\
\hline \multicolumn{6}{|c|}{$\begin{array}{l}\text { Positive means FXS in the first } 10 \\
\text { matches }\end{array}$} \\
\hline Statistic & Value & $95^{\circ}$ & & & \\
\hline Sensitivity & $100.00 \%$ & \multicolumn{4}{|c|}{$76.84 \%$ to $100.00 \%$} \\
\hline Specificity & $53.00 \%$ & \multicolumn{4}{|c|}{$42.76 \%$ to $63.06 \%$} \\
\hline Positive Likelihood Ratio & 2.13 & \multicolumn{4}{|c|}{$\begin{array}{l}1.73 \text { to } \\
2.62\end{array}$} \\
\hline Negative Likelihood Ratio & 0.00 & & & & \\
\hline Disease prevalence & $12.28 \%$ & \multicolumn{4}{|c|}{$6.88 \%$ to $19.75 \%$} \\
\hline Positive Predictive Value & $22.95 \%$ & \multicolumn{4}{|c|}{$19.48 \%$ to $26.84 \%$} \\
\hline Negative Predictive Value & $100.00 \%$ & & & & \\
\hline Accuracy & $58.77 \%$ & \multicolumn{4}{|c|}{$49.17 \%$ to $67.91 \%$} \\
\hline
\end{tabular}

\section{Figures}
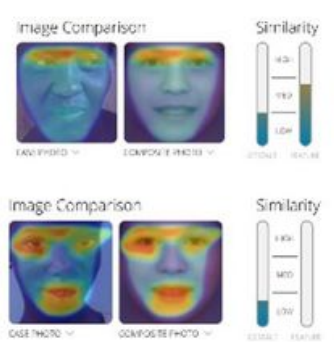

image Comparison

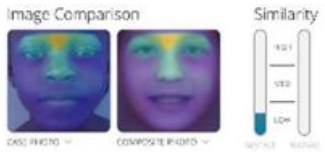

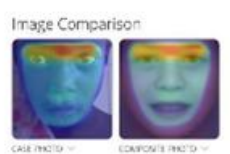

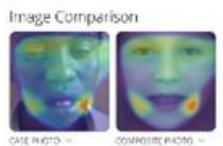

Image Comiarison

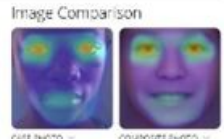

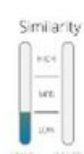
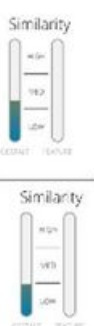

A
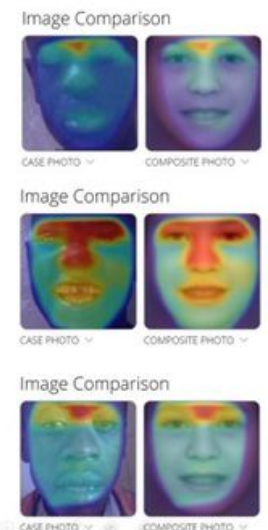
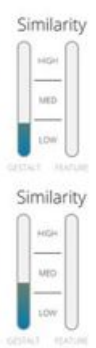

Similarity

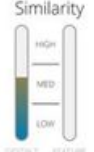

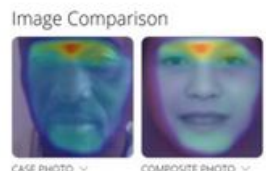
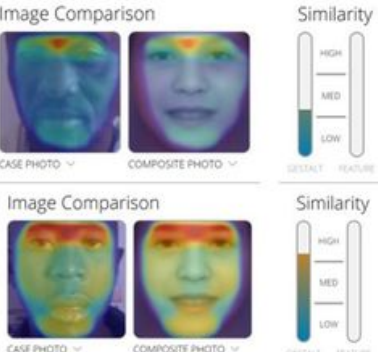

Image Comparison

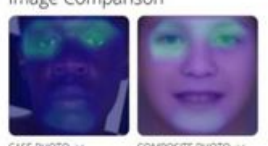


heat-maps and Fragile X syndrome masks of 12 FXS patients from this study.

$A$, female subjects and $B$, male subjects.

The red areas is where there is the most similitude with the syndrome mask

Score Distribution

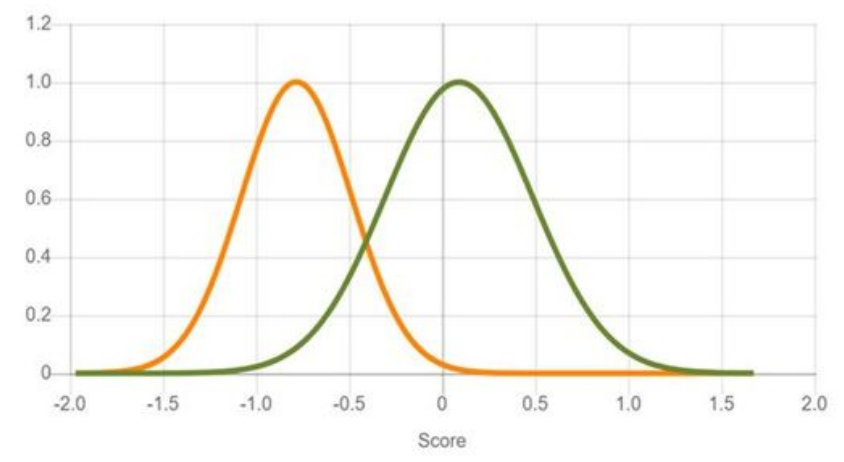

- FXS

- Non-FXS

Score Distribution

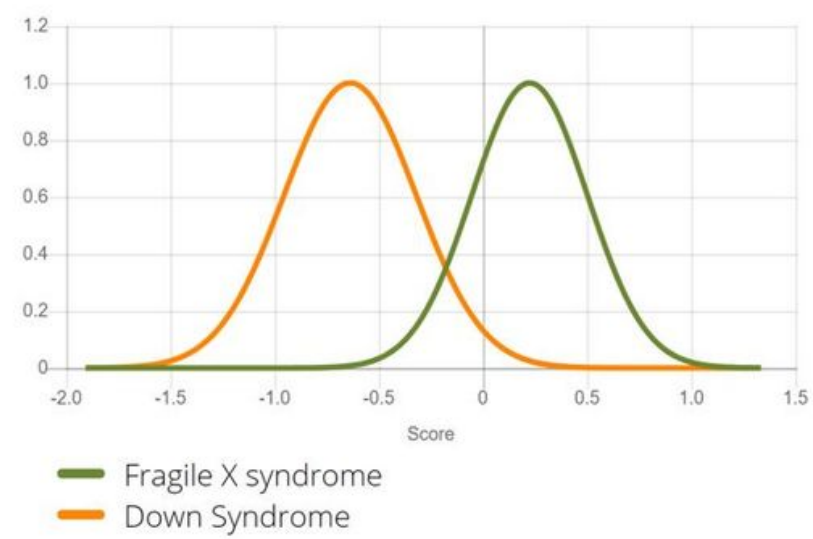

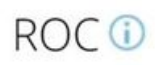

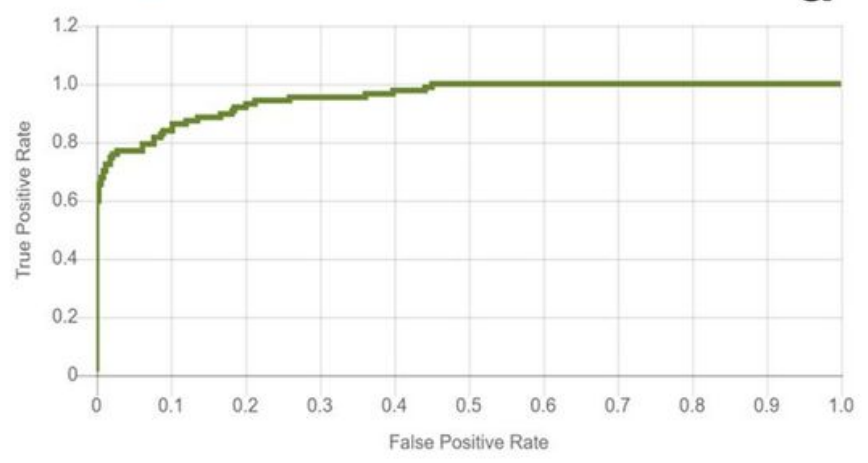

$\mathrm{AUC}=0.955$

P Value $=0.002$

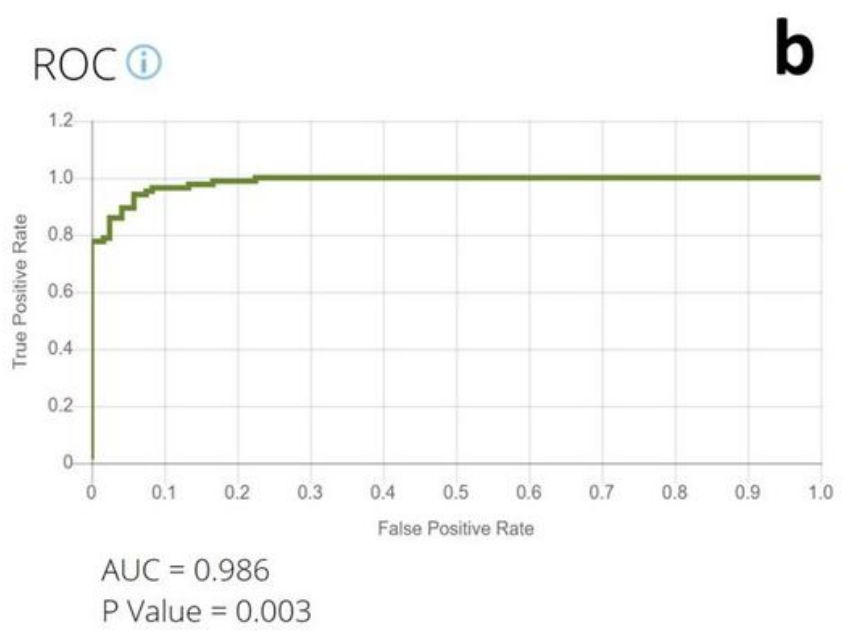

\section{Figure 2}

Binary comparison of Fragile $X$ subjects (FXS) versus Subjects with ID negative for Fragile $X$ syndrome (a); and Fragile $X$ subjects versus subjects with Down syndrome (b). 